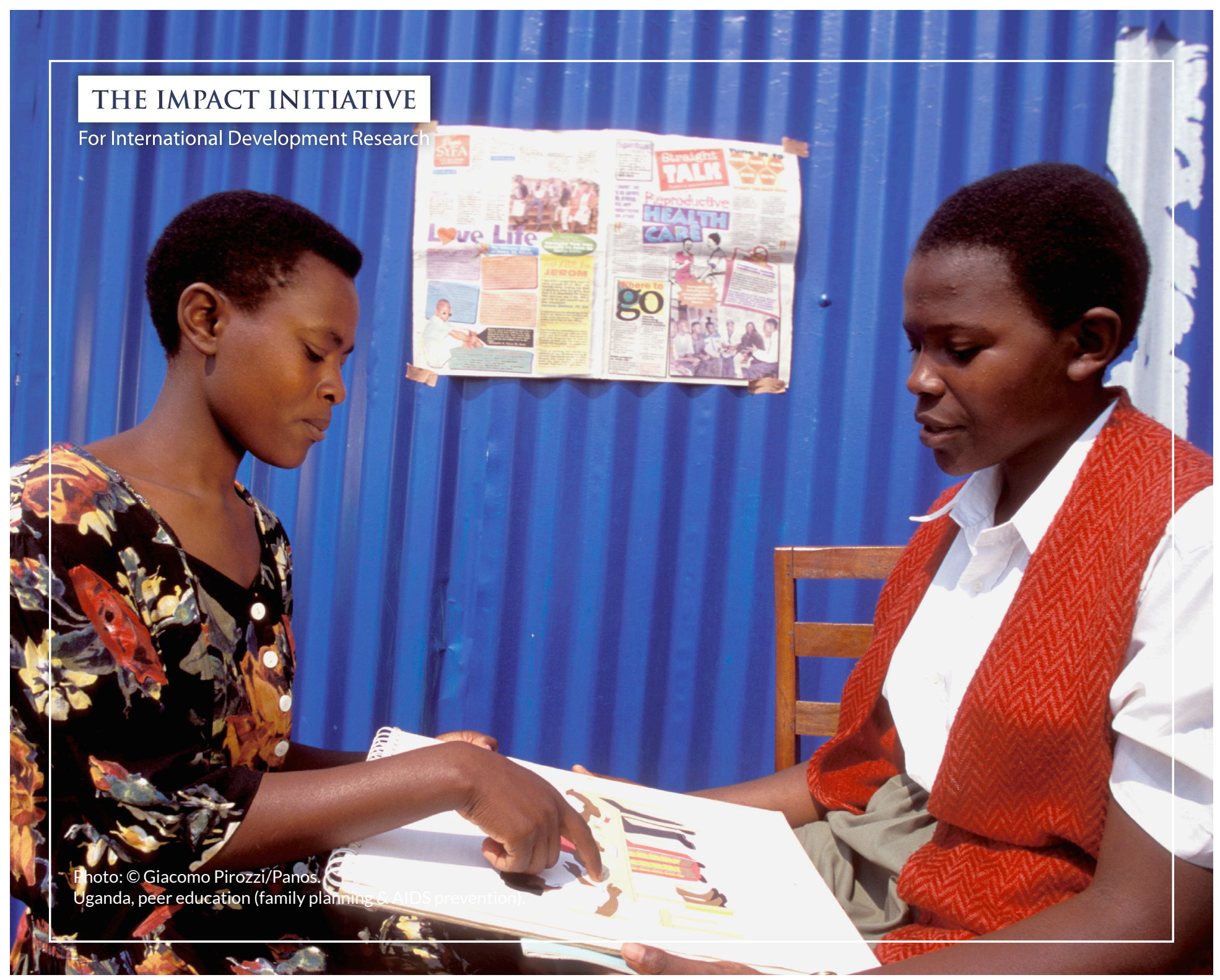

The Impact Lab // Learning Guides // Maximise mutual learning

\title{
Designing Programmes to Maximise Mutual Learning
}

Author: Emma Greengrass $\quad$ Published: November 2016

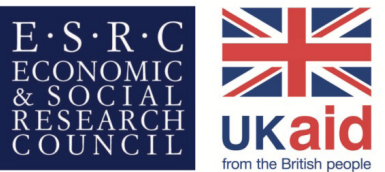

Research jointly supported by the ESRC and DFID 


\section{THE IMPACT INITIATIVE}

For International Development Research

The Impact Lab presents a series of Learning Guides which draw on the lessons for successful impact from grants funded by the ESRC-DFID Joint Fund for Poverty Alleviation Research. The Joint Fund aims to enhance the quality and impact of social science research, with the goal of reducing poverty amongst the poorest countries and peoples of the world. Since 2005, the Joint Fund has enabled over 150 research projects.

An impact evaluation, undertaken in 2015 , assesses the impact of the first two phases of the Joint Fund, and provides a thorough assessment of impact on policymakers, and other stakeholders over the ten years since it began. The evaluation, published in 2016, identifies critical barriers to engagement and uptake in areas like networks and relationships, mutual learning, individual capacities and incentives and lack of demand for evidence. Drawing on the ESRC's conceptual framework for impact assessment to inform the evaluation methodology, the evaluation also recognises the complexities of the research to policy process and the multifaceted nature of social science impact.

The Impact Lab seeks to strengthen links and create dialogue by providing an outline of relevant issues and clear lessons for knowledge practitioners, funders and researchers. Each Learning Guide, therefore, identifies replicable approaches to effective engagement in a particular area previously identified by the impact evaluation as a potential barrier for impact. Drawing on diverse case studies from the first two phases of the Joint Fund, this learning guide shares the strategies that have been successfully employed by ESRC DFID grant holders to increase outreach and maximise research uptake and impact in these critical areas. Many of these approaches may require a better understanding of local conditions, more time, effort or funding. However, the results could significantly strengthen the efficacy of research projects' pathways to impact. 


\section{Introduction}

Learning from research is a significant enabler of research impact that contributes to sustainable development ${ }^{3}$. Essentially, anyone involved in a research project can benefit from regular opportunities to come together to review learning arising from the project, and an exchange of relevant knowledge between different stakeholders can enrich project development. Once a project reaches completion, effective communication and dissemination strategies can ensure the lessons learnt are shared with a wider audience.

\section{What makes mutual learning difficult for development actors?}

- Lack of planning (and/or lack of flexibility to adapt the plan) for knowledge exchange to take place.

- Engagement with key individuals happens too late in the process (e.g. at dissemination stage) so that relevant shared experience is not captured early enough in (or is not captured throughout) the research cycle.

- Weak relationships with government, policymakers, research users, within the community, and/or with wider stakeholders can limit the ways that these key actors appreciate research findings.

- Differences of language, culture and context can make the process of shared learning more difficult.

- The ideology of government ministers (i.e. if fixed) may prevent engagement even where empirical evidence supports a policy change and this can hamper research findings from contributing to instrumental impact through change of policy or practice.
- Policymakers may not directly cite research making it difficult for researchers to establish how effective knowledge exchange and influence has been.

- Policymakers may not understand the language used by researchers.

- Research beneficiaries/subjects may be alienated by research they do not perceive a need for, and by policy or practice changes that they have had no stake in.

- Fostering meaningful exchanges between stakeholders, and supporting capacity building, can be time-consuming - competing research or research funder priorities may limit the potential for capacity building to develop.

Mutual learning is an iterative process that cannot be predicted and which requires time and space within research activities in order to be fruitful. This can be difficult to drive forward in a sustainable manner, particularly if it has not been conceived as part of the design or given attention in planning or evaluation. The openness and attitude of stakeholders is a critical factor for learning from research processes to be effective, as is the development of trust between individuals engaged in learning and research. Political and contextual barriers - such as whether policymakers and/or practitioners have an appetite for the research in the first place, or whether the intended beneficiaries of research (such as local communities) perceive a need for it - can hamper knowledge exchange and prevent research from having impact on policy or practice. Researchers may have a narrow definition of monitoring and evaluation (i.e. a narrow focus on outputs) which prevents them from keeping a track of the wider impact (or outcomes) of their work. 
Finally, time constraints and a lack of financial resources can limit the capacity for researchers to facilitate meaningful engagement through which lessons can be shared with a wider audience.

This Learning Guide aims to identify ways that mutual learning can be enabled. It highlights strategies and approaches that have resulted in learning and contributed to generating impact.

This Learning Guide draws on the lessons from the following projects funded by the UK's ESRC-DFID's Joint Fund for Poverty Alleviation Research: ${ }^{1}$

- Social movements and poverty ${ }^{4}$ (2007-10, Principal Investigator: Professor Anthony James Bebbington, University of Manchester) which explored the themes and knowledge gaps concerning how social movements can be an effective strategy for poor people to address their own poverty in Peru and South Africa. The project had significant conceptual impact - for example, influencing the inclusion of a social movements' component into the design of the DFID-funded Effective States and Inclusive Development (ESID) research programme (http://www.effective-states. org/). It also led to capacity building for the movement organisations involved in developing stronger strategies and identities.

- Inter-agency research on strengthening community based child protection for vulnerable children in Sierra Leone ${ }^{5}$ (2013-15, Principal Investigator: Professor Michael Wessells, Columbia University) which investigated how to strengthen child protection in Sierra Leone and aimed to reduce teenage pregnancy through community-led work on family planning, sexual and reproductive health education, and life skills. The project resulted in a reduction of teenage pregnancy rates. It also influenced Sierra Leone's Child and Family Welfare Policy through community-driven approaches.

- Shame, social exclusion and the effectiveness of anti-poverty programmes: a study in seven countries $^{6}$ (2010-12/13, Principal Investigator: Professor Robert Walker, University of Oxford) which took place across seven countries (rural Uganda and India, urban China, Pakistan, Korea and the United Kingdom (UK), and small town/urban Norway) explored the relationship between poverty, shame and exclusion, as a universal phenomenon. The project resulted in conceptual influence such as developing new empirical evidence, as well as instrumental influence, for example a workshop convened as part of the research influenced the introduction of an amendment to the International Labour Organization (ILO) Recommendation 202 on social protection to include 'respect for the rights and dignity of people covered by the social security guarantees'. 
- Improving educational evaluation and quality in China (IEEQC) ${ }^{7}$ (2008-10, Principal Investigator: Professor Sally Thomas, University of Bristol); and Improving teacher development and educational quality in China ITDEQC) $^{8} \quad(2010-14$, Principal Investigator: Professor Sally Thomas, University of Bristol). The first project, the IEEQC, investigated the nature and extent of school effectiveness in China through local-level application of quantitative evaluation methodologies (known as multilevel modelling) in rural and urban schools. The second project, ITDEQC, enriched professional teaching development and learning in China and explored how professional learning communities could be applied in the Chinese context. Both projects successfully developed the capacity of the in-country researchers in technical skills (methodologies, approaches) and confidence. 


\section{Top tips for researchers}

\section{1}

\section{Engage all stakeholders in learning early-on and throughout the life of the research project}

All five projects identified key stakeholders and established user engagement approaches. User engagement was critical for ensuring that the research achieved influence. Engaging stakeholders in learning early-on (i.e. not just at the dissemination phase) and in collaborative ways helps to build a culture of trust and openness between different stakeholders. Involving government also maximises the likelihood of getting ministers' buy-in and collaboration. When user engagement is ongoing and takes place regularly, influential stakeholders are more likely to have the messages they need, and be motivated to make positive changes. Developing a clear, targeted user engagement strategy can support relevant shared experience to emerge. Engaging researchers, policymakers, and community members in co-learning, so that each plays an active role in the process, greatly enriches the research.

Example: Inter-agency research on strengthening community based child protection for vulnerable children in Sierra Leone ${ }^{5}$

The community-based child protection project in Sierra Leone was successful in both range and depth of impacts. Researchers engaged stakeholders at a national level, continuously and from the outset. Early, collaborative engagement led to buyin and a sense of ownership - influential stakeholders saw this as an opportunity to come together to explore how to strengthen policy and practice in this area.

\section{'Because they were brought in, and this wasn't imposed from the outside but they were invited in, they saw it as an opportunity to learn more, and maybe to do it better'. \\ Professor Michael Wessells, Columbia University, Principal Investigator.}




\section{Planning for impact at the design stage can benefit user engagement strategies to utilise learning later on}

Impact strategy and planning can be an effective way to involve stakeholders in learning processes at critical points, as well as for developing activities and approaches with stakeholders to foster knowledge exchange. All four studies developed a variety of dissemination and communications activities that drew on user engagement and mutual learning in different ways. In two studies, an impact plan was developed during the design phase and was found to be useful for developing effective user engagement strategies. One found that developing the impact strategy and plan helped to support dissemination activities.

The ESRC Impact Toolkit (http://www.esrc.ac.uk/research/impact-toolkit/) has sections on Developing Pathways to Impact and Developing a communications and impact strategy, advice on developing knowledge exchange as well as a section which provides examples of impact from ESRC funded projects.

Example: Shame, social exclusion and the effectiveness of anti-poverty programmes: a study in seven countries ${ }^{6}$

The project on shame and social exclusion achieved conceptual and capacitybuilding impacts and was reported by the research team as having more impact than anticipated. Developing an impact plan at the design stage helped to identify key stakeholders (policymakers) and approaches (policy workshops and public meetings), as well as identify dissemination plans for the specific countries involved. 
Take up 'windows of opportunity' to influence policymakers

Planning for impact can provide a 'road-map', or an outline of intended impacts and ways to achieve them; however, it is also important that plans are flexible and can be adapted in order for researchers to take up 'windows of opportunity' where these become apparent.

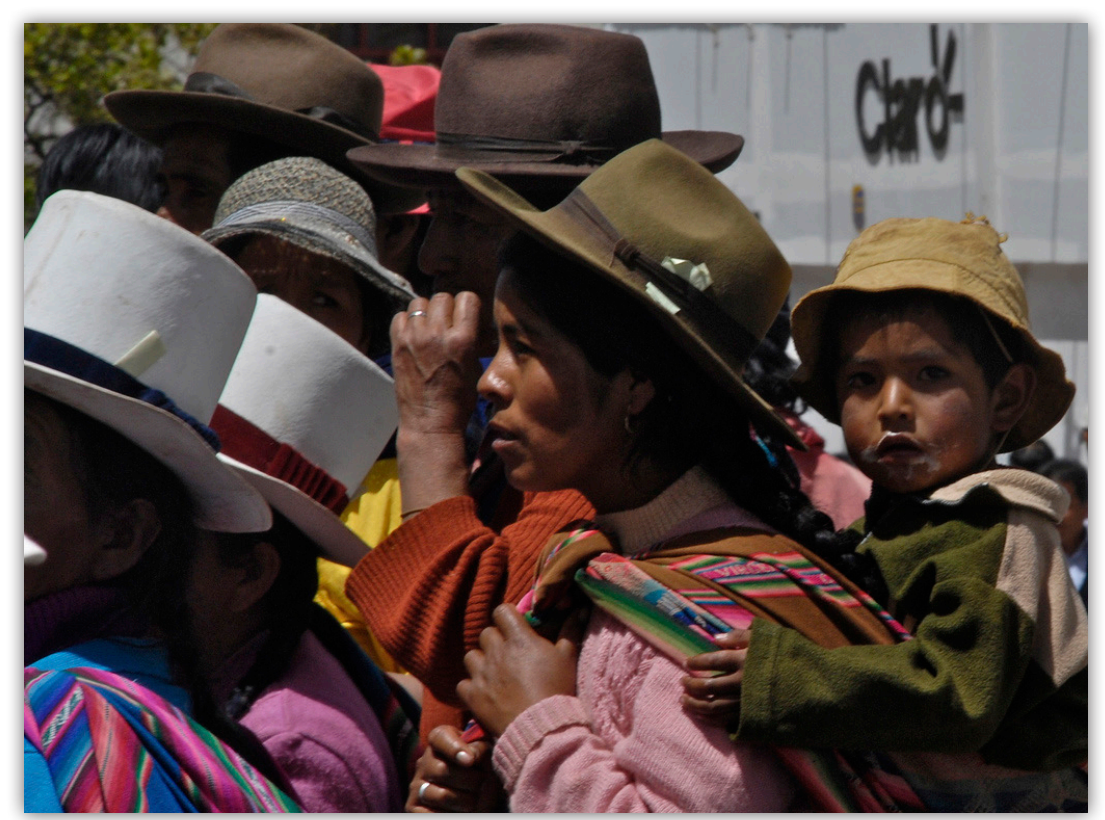

Photo: Rodrigoluca-foto/Flickr, licensed under CC BY-NC-ND 2.0.

\section{Example: Social movements and poverty ${ }^{4}$}

The Co-Investigator who led the South African research made the most of opportunities for discussion with the Director of Housing in Durban. Meetings took place before the research was funded, a few times during the course of the research, and were maintained for a few years once the project had ended. The Director supported some areas the Co-Investigator was advocating. Their discussions may have helped to influence policy change in line with recommendations arising from the research. Relationship-building is significant

- an ongoing conversation with an influential stakeholder may provide the evidence they need to support their arguments more effectively. Furthermore, discussion allows both researcher and policymaker to benefit from an exchange of findings and information. Although this can contribute to, or result in, policy changes it is hard to measure (particularly when conversations may take place informally), but taking up strategic opportunities when they arise can be a significant way to develop a two-way, ongoing conversation.

\section{'Making them [policymakers] more effective in terms of being able to push for policy changes.'}

Professor Dianna Mitlin, Manchester University, Co-Investigator (South Africa). 


\section{Collaborative, interactive events, workshops and training are conducive to stakeholder learning}

Each project coordinated events, workshops and/or training as part of user engagement, or dissemination, plans. The majority of these adopted collaborative, interactive and participatory approaches resulting in mutual learning between different stakeholders. Holding regular, ongoing workshops with stakeholders, where these are reflective, collaborative spaces, ensures that everyone contributes to how research is going and benefits from one another's knowledge, experience and understanding.

Example: Shame, social exclusion and the effectiveness of anti-poverty programmes: a study in seven countries ${ }^{6}$

The project on shame and social exclusion involved extensive knowledge exchange and adopted an inductive methodological approach and interactive, collaborative user engagement. A two-day workshop in Oxford, UK on 'Global Perspectives on the Experience of Poverty' brought together a range of potential research users (including influential international and national policy representatives) as well as non-governmental organisation (NGO) and media representatives. The workshop was highly interactive participants discussed research findings collectively, and collaborated with one another to develop ideas from the research findings for policy responses. The NGO and media representatives developed proposals for communicating the research. This led to a successful bid for additional funding for knowledge exchange activities from ESRC. A coconstructive approach was a significant factor in the success of the workshop, enabling key stakeholders to take an active role in developing and framing responses and communications. 
Involve intermediaries in the communication process with policymakers, and more widely

Dissemination and communication activities across all four studies included meetings, presentations, workshops, websites and publications. Two studies found knowledge intermediaries, or researchers acting as such, to be helpful in disseminating research findings to a wider audience. Intermediaries can also effectively support the process of knowledge exchange between researchers and policymakers. Policymakers may not understand the language of researchers whilst researchers may not possess the right skills for communicating research. Knowledge intermediaries, however, are well placed to bring research to policymakers (and to wider audiences) since their role is to bring producers and users of knowledge together, therefore helping to connect evidence with demand.

Example: Improving educational evaluation and quality in $\mathrm{China}^{7}$

In this project, in-country researchers (who were also members of the policy committees and other groups) supported the communication of research and played an 'intermediary' role in ensuring findings were translated to influential policymakers and practitioners in key institutions (such as the Ministry of Education and Local Education Authorities) in China. The use of 'intermediaries' in this project contributed to the debates emerging within policy circles.

\section{|'[The intention was] to spread a seed and keep encouraging others to make it grow'}

Dr Wen Jung, University of Bristol, Co-Investigator. 


\section{Capacity building transfers valuable skills and builds confidence}

Capacity building (developing technological skills and building confidence) is a crosscutting element in all four studies. Adequate time and funding for capacity building are critical factors for success in this area. Capacity building can require intensive periods of commitment and/or ongoing support in order to achieve sustainability in the medium- to longer term. Whilst funding may be limited, a well-designed intervention can result in the valuable transfer of skills and expertise, as was the case with two educational projects in China.

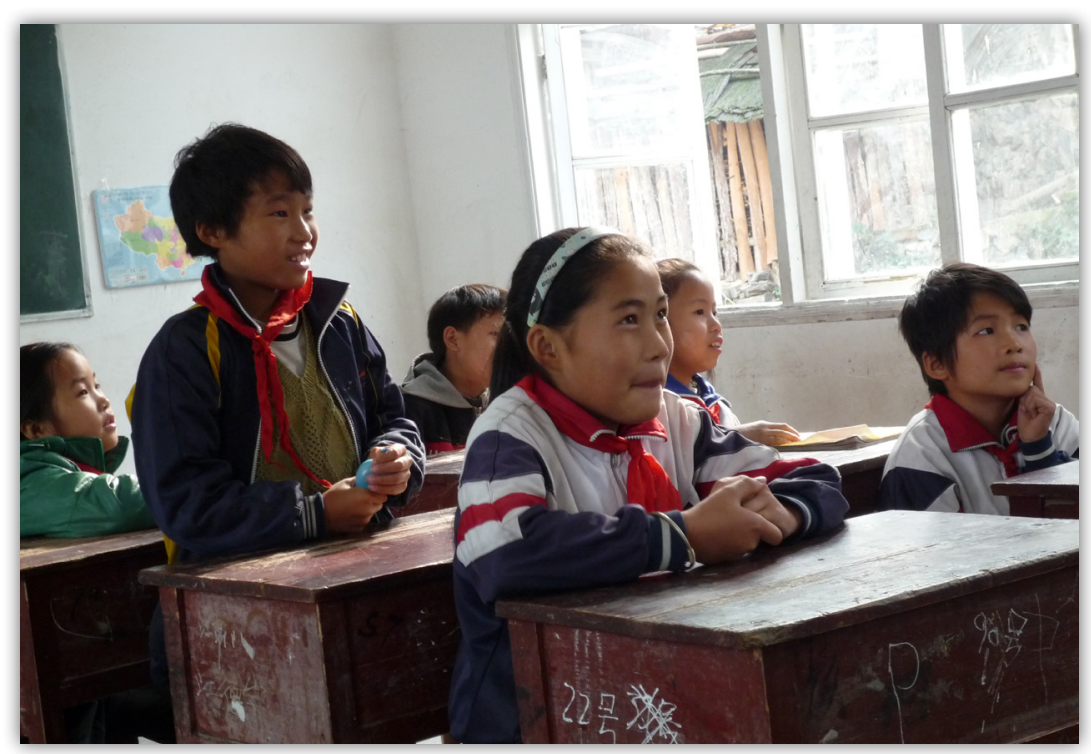

Baibi Mountain Village, Xijiang, Guizhou Province, China Photo: Thomas Galvez /Flickr, licensed under CC BY 2.0.

Example: Improving educational evaluation and quality in China ${ }^{7}$, and Improving teacher development and educational quality in China ${ }^{8}$

A major element of two educational projects in China involved intense capacity development. Beneficiaries for both projects were exposed to a range of methodologies and approaches, including: empirical research design; educational evaluation methodology; statistical analysis and qualitative analysis; as well as exploring enhanced notions of teacher development. The Southern-based researchers spent a three-month period undergoing training at the University of Bristol in the UK. This valuable transfer of skills supported the ongoing work of the researchers on return to their institutions, leading to promotion and scholarship opportunities for some, as well as benefiting the development of the research itself. The second of these projects also sought to explore whether the existing UK framework on developing professional communities (learning communities) in schools could be applied in the Chinese context. Follow-on funding (through the ESRC Impact Acceleration Awards) has been awarded for an impact study to improve the teaching materials and resources developed through the two projects and to further adapt them for Chinese teaching. 


\section{Utilise technology, creative media and social media for outreach}

Utilising technology and social media can support wider communications and outreach. One study, in particular, was fortunate in attracting additional funding (in the form of an ESRC grant to support knowledge exchange activities) allowing for a greater focus on the dissemination and communication of the research findings through creative media. Social media platforms also allow for a greater reach and for research findings to be communicated to, and taken up by, a wider audience (e.g. through blogging, and micro-blogging).

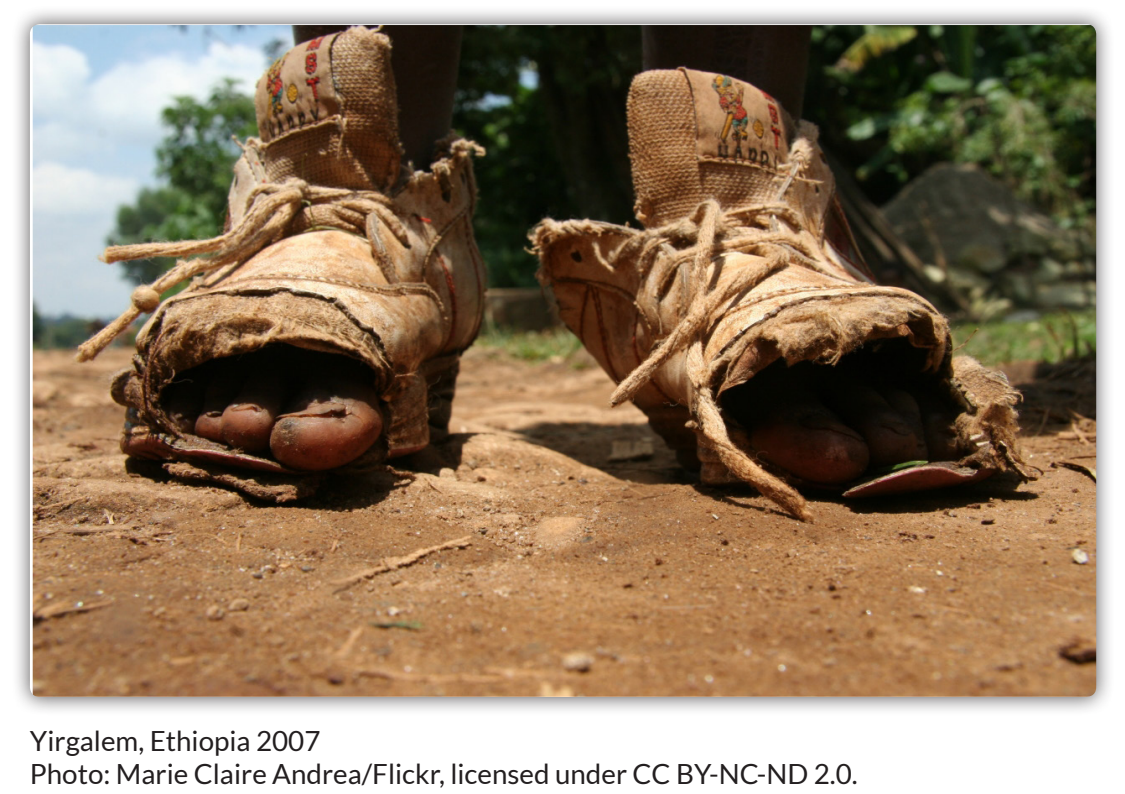

Example: Shame, social exclusion and the effectiveness of anti-poverty programmes: a study in seven countries ${ }^{6}$

The study on shame, social exclusion and poverty attracted additional funding that enabled work with technology and creative media to take place. This included: a play (developed by Pegasus Theatre) and an education pack for UK schools; a production company (Mediae) included storylines taken from the research in a soap opera ('Makutano Junction'), which ran in Kenya, Tanzania and Uganda; and a documentary ('Rich Man Poor Man' produced by Media Trust) was shown in the UK. These initiatives all drew on messages from the research, making learning more accessible for a wider audience. Communicating learning from social science or academic research in a creative fashion can effectively raise awareness, stimulate debate and change public attitudes. 


\section{Peer support networks and online support}

Online peer support and networking ${ }^{8}$ offers a cost-effective way for researchers and practitioners to develop spaces for mutual learning within a field of knowledge. Online Communities of Practice (CoP), where groups find commonality around a topic or field of expertise, are also good ways to develop research ideas and collaborative exchanges and can be very effective, particularly when individuals are spread out geographically. One of the capacity-building impacts arising from the study on shame and social exclusion has been the creation of a global peer support network, whilst the work on community-based child protection in Sierra Leone has led to the setting up of an online forum.

Example: Inter-agency research on strengthening community based child protection for vulnerable children in Sierra Leone ${ }^{5}$

The Community Child Protection Exchange is an online forum targeting practitioners, researchers and policymakers involved in community-based child protection work. This online forum brings key actors together to contribute to knowledge, policy and practice, and has been particularly effective at disseminating findings internationally. The forum was instrumental in producing a number of briefings to share research findings with practitioners in an accessible format. 


\section{Use participatory processes to define outcomes from the start}

Outcomes are the consequences of research in the medium- to longer term (as opposed to outputs which are related more to the immediate results). An evaluation of the ESRC-

DFID Joint Fund ${ }^{2}$ found that the majority of researchers had captured outputs and were less focused on looking at the outcomes for impact. It can be difficult to establish and capture outcomes, especially when funding is finite or monitoring and evaluation places a high emphasis on quantitative figures. However, capturing outcomes can provide evidence of longer-term impact of benefit to researchers, practitioners and funders. One study, in particular, had used a participatory process to define outcomes.

Example: Inter-agency research on strengthening community based child protection for vulnerable children in Sierra Leone ${ }^{5}$

Attention was given to outcomes at the outset. An ethnographic study found local people were not using existing systems of child protection as these were negatively perceived. The data from the ethnographic study was used to collate data on the main harms to children. A subsequent participatory methodology was then used to define outcomes with communities. Communities themselves drew up a framework around 'What does it mean to be a well child?' Outcomes were further refined through a population-based public health approach in order to influence national-level policy on outcomes related to harm, wellbeing, risk and protection factors. This participatory process helped to define outcomes but also supported locally driven concerns to shape nationally recognised child protection indicators in Sierra Leone. 
Advisory groups can be valuable for developing ongoing learning about context and strategy

Understanding the context for the research itself, as well as the context that policymakers are working within, is an important factor in developing effective plans for impact. An advisory group can be helpful in fostering understanding of context. In the study on social movements, the Co-Investigator involved in the research in South Africa felt that an advisory group would have been a valuable resource for the research.

Conversely, the work on education in China benefited from an advisory group at planning, dissemination and monitoring phases.
'...[an advisory group] tells you quite rapidly if
you've found things that are really very interesting that the policymakers haven't thought of before. And then it also helps because you immediately have people who are thinking about these issues who play your findings into other debates.'
Professor Dianna Mitlin, Manchester University, Co-Investigator (South Africa).

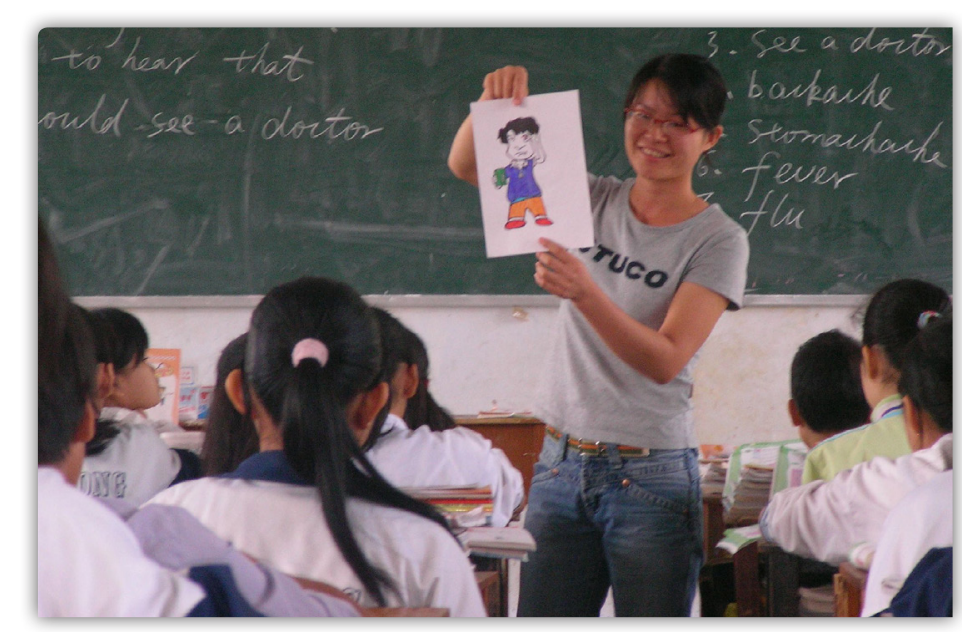

Student teacher, China.

Photo: Rex Pe/Flickr licensed under CC BY 2.0.

Example: Improving educational evaluation and quality in China ${ }^{7}$

The advisory group (which included national educational leaders and scholars), the research team and stakeholders met to develop communication with potential user groups so that these plans would be adapted for the context. The advisory group also held a key role in monitoring dissemination and research impact. 


\section{Top tips for research funders}

To support research that benefits from the shared learning of stakeholders, funders could consider the following:

\section{Provide additional funding}

Capacity building is not a 'quick win' and requires a commitment in terms of time, human resources and funding. To effectively transfer technical skills and build confidence requires intensive or ongoing support - the benefits can be long-lasting but may not be immediately apparent. Dissemination and communication work can also benefit from follow-on funds to enlist those with the specific skills required.

Following a pilot phase (in 2013-2014), ESRC DFID's Impact and Engagement Scheme in 2015 provided follow on funding to researchers funded within Phase 2 of the Joint Fund. The scheme was designed to enable researchers to respond to emerging opportunities for knowledge exchange and research impact.

ESRC also provides Impact Acceleration Accounts (IAA) which are block awards made to research organisations to accelerate the impact of research. The IIA scheme is designed to respond (flexibly and rapidly) to promote knowledge exchange in key areas including: building relationships and networks with potential research users, facilitating the coproduction of knowledge, supporting culture change around knowledge and improving skills and capabilities in this area.

\section{2}

\section{Provide researchers with guidance on monitoring impact outcomes}

A requirement on reporting outcomes, along with guidance from funders in this area, would help to fill the skills gap. According to the impact evaluation ${ }^{1}$, researchers were generally focused on monitoring outputs (such as dissemination events or publications) rather than on outcomes. Moreover, when it came to evidencing capacity-building outcomes, a minority of projects were able to draw on in-depth assessments to evidence outcomes ${ }^{1}$. 


\section{Listen to communities about outcomes}

Involving local people in developing outcomes and in policy dialogue can result in national policy and practices that reflect the needs of the local community. The community-based child protection project in Sierra Leone ${ }^{5}$ shows how successful a community-led approach can be in enabling links with government processes. The Mobilising Knowledge for Development (MK4D) programme $^{10}$ also explored collaborative approaches and sought to involve southern partners in the coconstruction of outputs such as the 'Training toolkit: the monitoring and evaluation for information literacy training initiative in Africa: a journey approach'.11 \& ${ }^{12}$

ESRC's Impact Acceleration Accounts (IAA) also enable researchers to build networks and relationships with potential research users and beneficiaries, to foster co-production processes, and improve engagement with wider stakeholders including civil society and local business.

\section{4}

\section{Be involved in collaborative problem-solving:}

Processes of collaborative problem-solving that involve research funders, researchers, policymakers and communities involved contribute to learning and impact. The Mobilising Knowledge for Development (MK4D) programme ${ }^{10}$ focused on strengthening knowledge exchange between different stakeholders to maximise the reach and impact of research. The programme was designed with a strong co-constructive and collaborative approach. The annual report to 31st March $2012^{11}$ highlights the co-production initiatives with key stakeholders, including Southern partners and donors, which were undertaken as part of the programme. When stakeholders are involved collaboratively in addressing a problem, solutions are more likely to build on existing knowledge. 


\section{Spotlight}

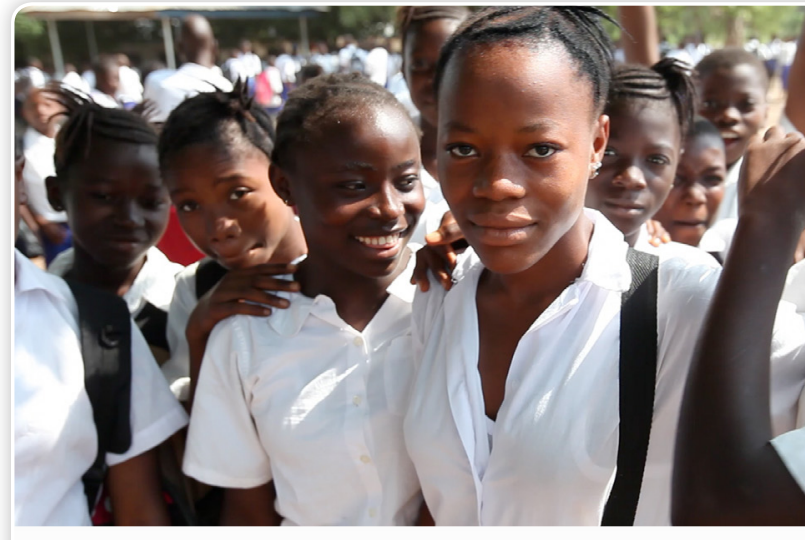

Strengthening community based child protection in Sierra Leone

The Inter-Agency Research on Strengthening Community Based Child Protection for Vulnerable Children in Sierra Leone ${ }^{5}$ project focused on strengthening child protection practice in Sierra Leone through community-driven action linking communities with aspects of the formal protection system. The intervention developed measures of children's protection and well-being, and aimed to reduce teenage pregnancy through community-led education.

\section{'The spirit of co-learning really influenced the relationship of learning with policymakers. This was an open process and a key enabler of discussions with stakeholders and with policymakers... So more of an approach of needing to learn more together around what are the problems and interventions needed as identified by local communities themselves.'}

\section{Professor Michael Wessells, Columbia University, Principal Investigator.}

The Inter-agency research on strengthening community based child protection for vulnerable children in Sierra Leone adopted a co-learning, participatory approach throughout. The project continues to influence nationally and internationally. The project has achieved the following impacts:

- Instrumental impact: Influenced a new Child and Family Welfare Policy with a community-driven approach using population-based measures of risk and wellbeing outcomes. The community led approach was approved by the Ministry of Social Welfare, Gender and Children's Affairs (MSWGCA) and made central in the new Child and Family Welfare Policy. and by the Cabinet. At the start of the project, child protection legislation in Sierra Leone adopted a top-down approach, alienating local communities. 
- Conceptual impact: Led to new thinking about how community-led approaches in child protection can be a significant enabler of links with government processes and effectively contribute to the development of formal systems, policies and practice. The research approaches (community-led, participatory action research) are being extended to other countries and there are plans to contextualise this work.

- Capacity-building impact: Research training provided for the national research team, and ongoing capacity building for child protection workers in Sierra Leone, contributed to the sustainability of the project.

- Poverty reduction impact: A reduction in teenage pregnancies in Sierra Leone, and local communities becoming connected up to the formal health system in a more holistic way.

\section{What factors enabled the impact?}

The research in Sierra Leone illustrates how collaborative processes that foster colearning with all stakeholders, and that are incorporated into project design and approaches, can benefit research outcomes that contribute to greater impact and sustainability.

1. Spirit of co-learning: What the Principal Investigator described as 'a spirit of colearning' was a strong enabler of the discussions at every level. Stakeholders included: the researchers, policymakers, government officials, NGOs, international non-governmental organisations (INGOs), partners, and the local communities involved. This was an inclusive process in which there was a mutual recognition of the need to come together to explore what the problems were and to identify solutions collectively. Project partners and NGO partners supported the researchers in developing this collaborative approach. Researchers did not arrive with pre-existing ideas of the scope or nature of the problem - these understandings were developed collectively and resulted in research that met an existing need. Ethnographic research was undertaken to clarify the child protection context in Sierra Leone - this contributed to bringing the Ministry on board. The National Child Protection Committee provided valuable knowledge of rural areas and helped to identify where to locate the research. Policymakers were receptive to learning from other stakeholders and from community-led approaches, which helped a valuable, mutual process to develop. The government, NGOs and INGOs co-constructed a shared understanding of policy and practice in child protection systems. Researchers (including one that was seconded from UNICEF) were instrumental in facilitating this engagement at all levels (including with the ministers and local chiefs). 
2. Community-driven approach: The project was designed from the start to involve community members in the process as active collaborators. Local people were involved in defining the problems and the needs of the community, in developing solutions, and in mediating with policymakers about new (bottom-up) policy in this area. Eventually this culminated in national policy changes that reflected community concerns (and replaced a top-down system that had previously alienated local people). Involving communities in the process of identifying outcomes, which were then taken up in national-level discussions of childhood harm, wellbeing, risk and protection, led to a greater sense of ownership of policy developments at the local level.

3. Role of the 'action research facilitator': The role of the researcher was redefined as an enabler of this collaborative approach. The Principal Investigator described this role as being 'a action research facilitator' working alongside the community to support their direct involvement in influencing policymakers, and talked about coming to the process as a 'co-learner' in the same way as all the other stakeholders involved in developing the project.

4. Connectivity - working alongside others to benefit from their knowledge: The project connected up with existing inter-agency knowledge. This helped to ensure that the choice of country was appropriate, the policy context was understood, and the timing for the research was good. By working with the Inter-Agency Learning Initiative on Strengthening Community-Based Child Protection Mechanisms and the Child Protection Systems Global Reference Group, and through UNICEF - a key agency working on child protection policy in Sierra Leone - it was possible to determine the clear demand within Sierra Leone for help in this area. This collaboration also provided confirmation of the timely nature of the project since a number of stakeholders (including UNICEF, the Ministry of Social Welfare, Gender and Children's Affairs (MSWGCA) as well as the Child Protection Committee) were aware that the existing system was not working effectively. UNICEF in Sierra Leone became strong allies, instrumental as relationship builders and power brokers. UNICEF also engaged mid-level ministers in the co-learning aspects of the project. Everyone, therefore, brought their knowledge to the table, ensuring the project was stronger as a result. This relationship-building, and knowledge sharing, positioned the project as part of wider ongoing inter-agency research in Sierra Leone (through the Inter-Agency Learning Initiative on Strengthening Community-Based Child Protection Mechanisms and Child Protection Systems). 


\section{Conclusion}

Mutual learning draws on the knowledge, experience and insights from all stakeholders involved, from the outset of a project and throughout, bringing individuals together to solve problems collectively. This collaborative process can help to ensure that research is well designed, is appropriate to the country and policy context, and that learning arising from the process is shared with influential stakeholders who are in a position to make legislative or policy changes. Engaging influential stakeholders in co-learning at the outset (for example, government ministers or departments) allows them to provide valuable inputs and establishes their buy-in. Involving local communities as active participants in shaping research can result in community-driven policy and practice.

\section{References and key resources}

\footnotetext{
${ }^{1} 2016$ Impact evaluation of the Joint Fund: http://www.theimpactinitiative.net/2016-impactevaluation-joint-fund
}

${ }^{2}$ ESRC What is Impact? http://www.esrc.ac.uk/research/impact-toolkit/what-is-impact/

3 Constantine, J.; Bloom, G. and Shankland, A. (2016) 'Towards Mutual Learning with the Rising Powers', IDS Policy Briefing 123, Brighton: IDS, https://opendocs.ids.ac.uk/opendocs/handle/123456789/12188

4 Social Movements and Poverty (2007-10, Principal Investigator: Professor Anthony James Bebbington, University of Manchester), www.theimpactinitiative.net/project/social-movementsand-poverty

${ }^{5}$ Inter-Agency Research on Strengthening Community Based Child Protection for Vulnerable Children in Sierra Leone (2013-15, Principal Investigator: Professor Michael Wessells, University of Columbia), www.theimpactinitiative.net/project/inter-agency-research-strengtheningcommunity-based-child-protection-vulnerable-children

6 Shame, Social Exclusion and the Effectiveness of Anti-Poverty Programmes: A Study in Seven Countries (2010-12/13, Principal Investigator: Professor Robert Walker, University of Oxford), www.theimpactinitiative.net/project/shame-social-exclusion-and-effectiveness-anti-povertyprogrammes-study-seven-countries

7 Improving Educational Evaluation and Quality in China (IEEQC) (2008-10, Principal Investigator: Professor Sally Thomas, University of Bristol), www.theimpactinitiative.net/project/improvingeducational-evaluation-and-quality-china

8 Improving Teacher Development and Educational Quality in China (ITDEQC) (2010-14,

Principal Investigator: Professor Sally Thomas, University of Bristol), www.theimpactinitiative. net/project/improving-teacher-development-and-educational-quality-china-examining-schoolsprofessional 
9 A Directory of Online Communities? An Experiment to Better Signpost CoPs to Internet Users, http://community.eldis.org/category/blog/

${ }^{10}$ Mobilising Knowledge for Development programme exceeds funder expectations. http:// www.ids.ac.uk/news/mobilising-knowledge-for-development-programme-exceeds-funderexpectations

${ }^{11}$ Mobilising Knowledge for Development Phase 2: Annual Report to 31st March 2012. http:// webarchive.nationalarchives.gov.uk/20160323135854/http://r4d.dfid.gov.uk/PDF/Outputs/ IDS/2012-MK4D-Annual-review-report-FINAL.pdf

12 Training toolkit: The monitoring and evaluation for information literacy training initiatives in Africa: a journey approach. https://opendocs.ids.ac.uk/opendocs/bitstream/ handle/123456789/2893/6758_IDS_toolkit_Interactive.pdf?sequence=1\&isAllowed=y

\section{Key resources}

Research funding and guidance:

- $\quad$ ESRC DFID Joint Fund for Poverty Alleviation Research Programme: http://www.esrc.ac.uk/research/international-research/international-development/esrcdfid-joint-fund-for-poverty-alleviation-research/

- $\quad$ ESRC DFID Raising Learning Outcomes in Education Systems Research Programme: http://www.esrc.ac.uk/research/international-research/international-development/esrcdfid-raising-learning-outcomes-in-education-systems-research-programme/

- $\quad$ ESRC Funding - information about funding opportunities and related guidance: http://www.esrc.ac.uk/funding/

- $\quad$ ESRC Impact Acceleration Accounts: http://www.esrc.ac.uk/funding/funding-opportunities/impact-acceleration-accounts/

- $\quad$ ESRC Impact Prize:

http://www.esrc.ac.uk/research/celebrating-impact-prize/

- $\quad$ ESRC Research Funding Guide - May 2016

http://www.esrc.ac.uk/files/funding/guidance-for-applicants/research-funding-guide/

Tools and guidance for building impact:

- $\quad$ DFID Research Uptake Guidance - published May 2013 (updated April 2016): https://www.gov.uk/government/publications/research-uptake-guidance

- $\quad$ ESRC Developing impact evaluation: http://www.esrc.ac.uk/research/research-and-impact-evaluation/developing-impactevaluation/ 
- ESRC DFID Joint Fund for Poverty Alleviation Research - Impact and Engagement scheme 2015

http://www.esrc.ac.uk/funding/funding-opportunities/esrc-dfid-impact-and-engagementscheme-2015/

- $\quad$ ESRC Impact Case Studies:

http://www.esrc.ac.uk/news-events-and-publications/impact-case-studies/

- $\quad$ ESRC Impact Toolkit: provides definitions of impact; guidance and support for maximizing research impact; for 'Developing Your Pathway to Impact' (http://www.esrc.ac.uk/research/ impact-toolkit/developing-pathways-to-impact/); and includes a variety of communications tools for developing effective research communications:

http://www.esrc.ac.uk/research/impact-toolkit/

- $\quad$ ESRC 'Pathways to Impact for Je-S (Joint Electronic Submission System) applications guidance for applicants:

http://www.esrc.ac.uk/funding/guidance-for-applicants/je-s-electronic-applications/ pathways-to-impact-for-je-s-applications/

- The UK Collaborative on Development Sciences (UKCDS): provide a useful guide on Finding and Building Effective Partnerships (http://www.ukcds.org.uk/resources/finding-andbuilding-effective-partnerships) along with a range of resources on relationship building and collaborative working: http://www.ukcds.org.uk/resources

Further resources:

- $\quad$ Evaluating the Impact of the ESRC-DFID Joint Fund for Poverty Alleviation Research: Final report to ESRC and DFID (March 2016)

http://www.esrc.ac.uk/files/research/research-and-impact-evaluation/evaluating-theimpact-of-the-esrc-dfid-joint-fund-for-poverty-alleviation-research/

- $\quad$ Related to this report: The Joint Fund for Poverty Alleviation Research impact evaluation: a response from ESRC and DFID (March 2016):

http://www.esrc.ac.uk/files/research/research-and-impact-evaluation/joint-fund-forpoverty-alleviation-research-impact-evaluation-a-response-from-dfid-and-esrc/

- $\quad$ Policy, practice and business impacts: evaluation http://www.esrc.ac.uk/research/research-and-impact-evaluation/policy-practice-andbusiness-impacts-evaluation-studies/ 


\section{Capacity Building*}

Through technical and personal skill development

\section{Co-construction (of knowledge)}

An approach to learning in which the focus is on collaborating with others in order to build a body of knowledge and understanding that is shared by everyone in the group - individuals are actively involved in the process of developing understanding as equal partners.

\section{Co-learning}

Collaborative learning in which individuals come together (either as pairs or as a larger group) to capitalize on one another's experience, skills, and perspectives in order to develop a common understanding.

\section{Co-production}

Collaborative and reciprocal process by which individuals design, develop and deliver a product (the research, or research outputs such as a publication, event or workshop) through equal partnership.

\section{Communication pathways}

A method or strategy that engages those with knowledge and ensures that information is effectively communicated to a wider audience.

\section{Communities of Practice (CoP)}

Where individuals interact as a group around a common theme, topic or body of knowledge in order to exchange learning and understanding. Online Communities of Practice can be useful forums of peer support, particularly when individuals are spread geographically.

\section{Conceptual*}

Contributing to the understanding of policy issues, reframing debates

\section{Cumulative influence*}

Research impact and influence that emerges over a longer period of time as evidence and debate increases, grows and deepens.

\section{Instrumental *}

Influencing the development of policy, practice or service provision, shaping legislation, altering behaviour

\section{Knowledge broker}

"A knowledge broker is an intermediary (an organization or a person), that aims to develop relationships and networks with, among, and between producers and users of knowledge by providing linkages, knowledge sources, and in some cases knowledge itself..." (Wikipedia)

\section{Knowledge exchange}

Knowledge exchange is a process that brings all stakeholders together (i.e. researchers, research users, policy-makers, and communities) in order to exchange expertise, information, ideas, experience and to learn from learning emerging from research.

\section{Knowledge exchange capacity}

Developing the skills and ability to foster knowledge exchange.

\section{Knowledge intermediaries}

The knowledge intermediary role is to bring producers and users of knowledge together therefore helping to connect evidence with demand.

\section{Mutual learning}

Process of collaborative learning between two or more individuals. A broad definition of mutual learning in a research context would include all stakeholders being engaged in collective learning from research from the outset and continuously throughout in order to benefit the development of the research and support its' medium to longer term impact and sustainability. Mutual learning can also be applied to the communication and dissemination of lessons learnt to a wider audience.

\section{Outputs}

Outputs are related more to the immediate results of research in terms of what was produced or undertaken.

\section{Outcomes}

Outcomes are the consequences of research in the medium to longer term.

*These definitions are drawn from the following resources:

- What is impact? The Economic and Social Research Council (ESRC) Toolkit

- Evaluating the Impact of the ESRC-DFID Joint Fund for Poverty Alleviation Research. 


\section{THE IMPACT INITIATIVE}

For International Development Research

The Impact Initiative for International Development Research exists to increase the uptake and impact of two programmes of research funded through the ESRC-DFID Strategic Partnership. These are: (i) The Joint Fund for Poverty Alleviation Research, and (ii) The Raising Learning Outcomes in Education Systems programme. The Initiative helps identify synergies between these programmes and their grant holders, and supports them to exploit influencing and engagement opportunities and facilitates mutual learning.

The Impact Initiative is a collaboration between the Institute of Development Studies (IDS) and the University of Cambridge's Research for Equitable Access and Learning (REAL) Centre.

www.theimpactinitiative.net

All content is available under the Open Government License v3.0, except where otherwise stated.
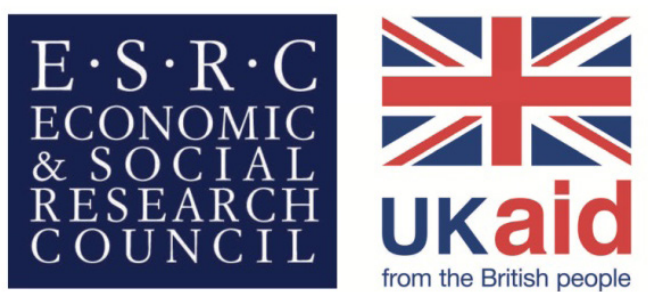

Research jointly supported by the ESRC and DFID 